\title{
MR-proANP and incident cardiovascular disease in patients with type 2 diabetes with and without heart failure with preserved ejection fraction
}

Jesper Jensen ${ }^{1,6^{*}} \mathbb{D}$, Morten Schou ${ }^{1,6}$, Caroline Kistorp ${ }^{2,6}$, Jens Faber ${ }^{3,6}$, Tine W. Hansen ${ }^{4}$, Magnus T. Jensen ${ }^{5}$, Henrik U. Andersen ${ }^{4}$, Peter Rossing ${ }^{4,6}$, Tina Vilsbø $\|^{4,6}$ and Peter G. Jørgensen ${ }^{1}$

\begin{abstract}
Background: Mid-regional pro-atrial natriuretic peptide (MR-proANP) is a useful biomarker in outpatients with type 2 diabetes (T2D) to diagnose heart failure (HF). Elevated B-type natriuretic peptides are included in the definition of HF with preserved ejection fraction (HFpEF) but little is known about the prognostic value of including A-type natriuretic peptides (MR-proANP) in the evaluation of patients with T2D.

Methods: We prospectively evaluated the risk of incident cardiovascular (CV) events in outpatients with T2D ( $n=806$, mean \pm standard deviation age $64 \pm 10$ years, 65\% male, median [interquartile range] duration of diabetes 12 [6-17] years, $17.5 \%$ with symptomatic HFpEF) according to MR-proANP levels and stratified according to HF-status including further stratification according to a prespecified cut-off level of MR-proANP.

Results: A total of $126 \mathrm{CV}$ events occurred (median follow-up 4.8 [4.1-5.3] years). An elevated MR-proANP, with a cutoff of $60 \mathrm{pmol} / \mathrm{I}$ or as a continuous variable, was associated with incident $\mathrm{CV}$ events $(\mathrm{p}<0.001)$. Compared to patients without HF, patients with HFpEF and high MR-proANP ( $\geq 60$ pmol/l; median 124 [89-202] pmol/l) and patients with $\mathrm{HF}$ and reduced ejection fraction (HFrEF) had a higher risk of CV events (multivariable model; hazard ratio (HR) 2.56 [95\% Cl 1.64-4.00] and 3.32 [1.64-6.74], respectively). Conversely, patients with HFpEF and low MR-proANP (<60 pmol//; median 46 [32-56] pmol/l) did not have an increased risk (HR 2.18 [0.78-6.14]).

Conclusions: Patients with T2D and HFpEF with high MR-proANP levels had an increased risk for CV events compared to patients with HFpEF without elevated MR-proANP and compared to patients without HF, supporting the use of MR-proANP in the definition of HFpEF from a prognostic point-of-view.
\end{abstract}

Keywords: Cardiovascular disease, Diabetes complications, Macrovascular disease, Type 2 diabetes, MR-proANP, Heart failure

*Correspondence: jesper.jensen.06@regionh.dk

${ }^{1}$ Department of Cardiology, Herlev and Gentofte Hospital, Borgmester Ib Juuls Vej 1, 2730 Herlev, Denmark

Full list of author information is available at the end of the article

\section{Background}

The development of heart failure (HF) in patients with type 2 diabetes (T2D) worsens prognosis dramatically [1]. Especially HF with preserved ejection fraction (HFPEF) is frequent in these patients [2]. However, the diagnosis of HFpEF is challenging, and more knowledge on HFpEF in patients with T2D is needed [3]. In the most recent HF guidelines from the European Society

(c) The Author(s) 2020. This article is licensed under a Creative Commons Attribution 4.0 International License, which permits use, sharing, adaptation, distribution and reproduction in any medium or format, as long as you give appropriate credit to the original author(s) and the source, provide a link to the Creative Commons licence, and indicate if changes were made. The images or other third party material in this article are included in the article's Creative Commons licence, unless indicated otherwise in a credit line to the material. If material is not included in the article's Creative Commons licence and your intended use is not permitted by statutory regulation or exceeds the permitted use, you will need to obtain permission directly from the copyright holder. To view a copy of this licence, visit http://creativeco mmons.org/licenses/by/4.0/. The Creative Commons Public Domain Dedication waiver (http://creativecommons.org/publicdomain/ zero/1.0/) applies to the data made available in this article, unless otherwise stated in a credit line to the data. 
of Cardiology (ESC), the diagnosis of HFpEF includes signs and/or symptoms of HF, echocardiographic criteria, and increased natriuretic peptides [4]. Natriuretic peptides are sensitive markers of hemodynamic status and elevated levels might represent an unstable state of $\mathrm{HFpEF}$, that is, a maladapted cardiac remodeling leading to subsequent cardiovascular (CV) events [5]. However, only B-type natriuretic peptides (B-type natriuretic peptide (BNP) and N-terminal proBNP (NT-proBNP)) are included in the HFpEF diagnosis and data on A-type natriuretic peptides (mid-regional pro-atrial natriuretic peptide (MR-proANP)) are lacking [6]. In a recent position paper from the ESC HF association, measurement of natriuretic peptides in high-risk populations such as patients with T2D was recommended, but again, evidence is primarily existing on B-type natriuretic peptides, and more data on A-type natriuretic peptides are needed [7]. A-type natriuretic peptides could carry similar relevant prognostic information when included in the HFpEF definition, as has previously been shown for B-type natriuretic peptides [4]. In the acute setting, MR-proANP has shown to improve the diagnostic performance of B-type natriuretic peptides for HF in obese patients [8]. Also, MR-proANP has been shown to be associated with clinical outcomes in HFpEF, not HF with reduced ejection fraction (HFrEF), [9] and in an at-risk community population, NT-proBNP and MR-proANP have shown to predict incident HF [10]. Moreover, MR-proANP levels have shown similar diagnostic performance as NT-proBNP in primary care patients suspected of non-acute HF [11]. And in a high-risk population such as outpatients with T2D, our group recently reported, that MR-proANP with a cut-off of less than $60 \mathrm{pmol} / \mathrm{l}$ was efficient to rule out HFrEF and at this cut-off, the highest ability to similarly rule out HFpEF was obtained. However, the accuracy to rule out HFpEF was still relatively low. Despite having a relatively low diagnostic value in HFpEF, the prognostic value of MR-proANP in HFpEF could potentially guide the diabetologist in identifying patients with a high risk of future clinical events, in which further cardiac work up should be performed and relevant treatment started [2]. In the present study, we sought to evaluate the prognostic value of MR-proANP and examine the significance of HFpEF in the presence and absence of elevated MRproANP levels using the pre-specified cut-off level.

\section{Methods}

The study was conducted in accordance with the Helsinki Declaration and approved by the Danish National Committee on Biomedical Research Ethics (amendment to protocol no. H-3-2009-139) [12].

\section{Study population}

The study population consisted of patients with T2D from the Thousand\&2 Study, which has previously been described in detail [13]. In brief, patients followed at two specialized diabetes clinics in Denmark, the Center for Diabetes research (CfD) at Herlev-Gentofte University Hospital and the Steno Diabetes Center Copenhagen (SDCC), were eligible to participate [13]. A total of 1030 patients were included in the Thousand\&2 Study (participation rate $47.8 \%$ ). Of these, two hundred and twentyfour patients with either prior heart valve replacement, atrial fibrillation and/or missing laboratory values/echocardiographic measurements were excluded rendering 806 patients available for the present study [2]

\section{Baseline study visit, biochemistry and echocardiograms}

The baseline study visit, biochemistry and echocardiograms have previously been described in detail $[2,14]$. In brief, all study related data were collected at or in close conjunction with the baseline study visit including blood samples for the study biobank and the echocardiographic assessments. After immediate centrifugation, blood samples were stored at $-80{ }^{\circ} \mathrm{C}$ and MR-proANP was analyzed in a single batch in July 2017 using the KRYPTOR assay (BRAHMS GmbH/Thermo Fisher Scientific, Hennigsdorf, Germany) [2]. Echocardiograms were primarily (>95\%) performed and analyzed by a single investigator (P.G.J.) in accordance with the existing European and American guidelines as previously described [14, 15]. Albuminuria was defined as urine albumin/creatinine ratio above $30 \mathrm{mg} / \mathrm{g}$ or urine albumin above $30 \mathrm{mg} /$ day on at least 2 consecutive measurements [13].

\section{Heart failure definitions}

Based on the current European and American guidelines we defined HF as follows: HFpEF was defined as selfreported dyspnea corresponding to the New York Heart Association (NYHA) functional class II-IV and presence of at least one of the following echocardiographic findings (a-d): (a) left ventricular ejection fraction (LVEF) $>40 \%$ and $\leq 50 \%$, (b) ratio of early diastolic mitral inflow velocity (E) to early diastolic septal annular velocity $\left(\mathrm{e}^{\prime}\right)\left(\mathrm{E} / \mathrm{e}_{\text {septal }}^{\prime}\right) \geq 15$, (c) increased left ventricular (LV) mass index $\left(>95 \mathrm{~g} / \mathrm{cm}^{2}\right.$ for women and $>115 \mathrm{~g} / \mathrm{cm}^{2}$ for men), and (d) left atrial volume index $>34 \mathrm{~mL} / \mathrm{m}^{2}$. HFrEF was defined as a $L V E F \leq 40 \%$, regardless of reported dyspnea. The definitions were specified before the MRproANP analyses were performed.

\section{Follow-up}

Information on incident $\mathrm{CV}$ events were retrieved through national registries. A CV event was defined as 
the composite of admission with CV disease (including $\mathrm{HF}$, coronary revascularization, myocardial infarction, cardiac arrest, cerebrovascular disease and peripheral artery disease) and CV death.

\section{Statistics}

Baseline characteristics for all four groups were compared using a one-way analysis of variance for continuous variables and, in case of non-normal distribution, MannWhitney U tests or Kruskall-Wallis tests were used. Categorical variables were compared using the Chi-square test. MR-proANP levels were non-normally distributed and, therefore, log2-transformed before continuous analyses. Cumulative incidence curves with non- $\mathrm{CV}$ death as competing risk for incident $\mathrm{CV}$ events according to HF status were performed, and for HFpEF also according to the prespecified dichotomized MR-proANP level of $60 \mathrm{pmol} / \mathrm{l}$. Moreover, a cumulative incidence curve for incident $\mathrm{CV}$ events according to the dichotomized MR-proANP level was performed with non-CV death as competing risk. Poisson regression with restricted cubic splines was used to examine the association of incidence rates and MR-proANP levels. The Akaike information criterion was used to determine the optimal number of knots. A multivariable Cox proportional hazards model was constructed including the prespecified variables age, sex, duration of diabetes, known CV disease, uncontrolled systolic blood pressure (SBP) above $170 \mathrm{mmHg}$, body mass index (BMI) and albuminuria. The performance of the multivariable model was assessed by C-statistics and continuous net reclassification index (NRI) with and without HF or MR-proANP in the model. Associations between MR-proANP levels and the echocardiographic measures were analyzed. A p-value less than 0.05 (two-sided) was considered significant. Statistics were calculated using $\mathrm{R}$ for Mac, version 3.4.2 ( $\mathrm{R}$ Project for Statistical Computing, Vienna University of Economics and Business administration, Austria).

\section{Results}

\section{Baseline characteristics}

Baseline characteristics for the 806 included patients are presented in Table 1. Compared to patients with HFpEF and a low MR-proANP, patients with HFpEF and a high MR-proANP were characterized by being older, with a higher frequency of males, a higher prevalence of known ischemic heart disease, a higher creatinine level and were to a higher extend treated with betablockers, diuretics and anti-platelet therapy. Generally, patients with HFpEF and a high MR-proANP were similar to patients with HFrEF. During a median [interquartile range (IQR)] follow-up of 4.8 [4.1-5.3] years, a total of 126 incident $\mathrm{CV}$ events occurred in the total cohort.

\section{Incidences of CV events according to HF status}

Patients with HFrEF, followed by patients with HFpEF and a high MR-proANP level, had the highest incidence of CV events (Fig. 1). Notably, patients with HFpEF and a low MR-proANP had an incidence of CV events, which was not statistically different to the incidence in patients without HF (Fig. 1). MR-proANP levels were associated with incident CV events; both analyzed as a dichotomized and a continuous variable (Fig. 2). In the univariable analysis and in the multivariable model, a higher MR-proANP level was associated with a higher risk of an incident $\mathrm{CV}$ event, with a hazard ratio (HR) [95\% confidence interval (CI)] of 2.09 [1.73-2.52] and 1.71 [1.34-2.18] per doubling of MR-proANP, $\mathrm{p}<0.001$, respectively (Fig. 3). Patients with HFpEF and a low MR-proANP did not have a significantly different risk for an incident $\mathrm{CV}$ event compared to patients without HF (univariable analysis; HR 1.30 [0.48-3.56], multivariable model; HR 2.18 [0.78-6.14], p >0.05) (Fig. 3). In contrast, the risk was significantly increased in both patients with HFpEF and high MR-proANP (univariable analysis; HR 3.47 [2.34-5.13], multivariable model; HR 2.56 [1.64-4.00], p < 0.001) and in patients with HFrEF (univariable analysis; HR 6.25 [3.23-12.11], multivariable model; HR 3.32 [1.64-6.74], p<0.001) (Fig. 3). A sensitivity analysis with adjustment for SBP as a continuous variable instead of adjustment for SBP $>170 \mathrm{mmHg}$ and with further adjustment for estimated glomerular filtration rate (eGFR) in addition to the prespecified variables showed overall similar results. Similarly, a sensitivity with adjustment for HbA1c instead of duration of type 2 diabetes in the prespecified model, also showed overall similar results (data not shown). In a competing risks regression model with the prespecified variables, sub-hazard ratios showed similar results as the traditional hazard ratios (Additional file 1: Table S1).

\section{Model performance}

Results of the prognostic performance of models with MR-proANP and HF status alone and added to the multivariable adjusted model are shown Table 2. An MR-proANP level with a cut-off of $60 \mathrm{pmol} / \mathrm{l}$ had only limited prognostic performance assessed by c-statistics, 0.57 (0.54-0.62). MR-proANP as a continuous variable had a c-statistic of $0.66(0.61-0.71)$, which was lower than the multivariable adjusted model alone, 0.72 (0.68-0.78). Adding MR-proANP as a continuous variable to the multivariable model, resulted in a significant 
Table 1 Baseline characteristics according to heart failure status

\begin{tabular}{|c|c|c|c|c|c|}
\hline & No HF & HFpEF low MR-proANP & HFpEF high MR-proANP & HFrEF & $\mathrm{p}$-value \\
\hline$n$ & 646 & 27 & 114 & 19 & \\
\hline Age, year (median [IQR]) & $64.4[56.9-69.8]$ & $60.2[53.0-65.7]$ & $69.8[64.7-74.9]$ & $72.2[64.9-76.6]$ & $<0.001$ \\
\hline Male, n (\%) & $433(67.0)$ & $10(37.0)$ & $67(58.8)$ & $15(78.9)$ & 0.003 \\
\hline Body mass index, $\mathrm{kg} / \mathrm{m}^{2}$ (median [IQR]) & $28.7[26.0-32.8]$ & $31.8[28.9-38.2]$ & $31.6[28.0-34.2]$ & $28.4[26.0-31.3]$ & $<0.001$ \\
\hline T2DM duration, year (median [IQR]) & $11.0[5.0-16.0]$ & $15.0[8.0-23.5]$ & $15.0[7.3-20.8]$ & $12.0[10.0-23.0]$ & $<0.001$ \\
\hline Known ischemic heart disease, $\mathrm{n}(\%)$ & $97(15.0)$ & $2(7.4)$ & $42(36.8)$ & $9(47.4)$ & $<0.001$ \\
\hline Smoking, n (\%) & & & & & 0.903 \\
\hline Never & $276(42.7)$ & $13(48.1)$ & $47(41.2)$ & $8(42.1)$ & \\
\hline Active & $88(13.6)$ & $5(18.5)$ & $19(16.7)$ & $2(10.5)$ & \\
\hline Former & $282(43.7)$ & $9(33.3)$ & $48(42.1)$ & $9(47.4)$ & \\
\hline Systolic blood pressure, $\mathrm{mmHg}($ mean (sd)) & $134.6(16.2)$ & $134.8(15.7)$ & $139.1(20.0)$ & $141.3(18.6)$ & 0.029 \\
\hline Diastolic blood pressure, mmHg (mean (sd)) & $80.0(10.8)$ & $79.8(10.1)$ & $77.8(10.9)$ & $81.6(9.3)$ & 0.226 \\
\hline Heart rate, bpm (mean (sd)) & $72.4(11.2)$ & $76.6(9.7)$ & $68.2(10.8)$ & $72.7(11.6)$ & $<0.001$ \\
\hline Angina, n (\%) & $44(7.0)$ & $6(22.2)$ & $23(20.7)$ & $4(22.2)$ & $<0.001$ \\
\hline Dyspnea, n (\%) & $162(25.1)$ & $27(100)$ & $114(100)$ & $8(42.1)$ & $<0.001$ \\
\hline New York Heart Association class, n (\%) & & & & & $<0.001$ \\
\hline । & $484(74.9)$ & $0(0.0)$ & $0(0.0)$ & $11(57.9)$ & \\
\hline$\|$ & $117(18.1)$ & $13(48.1)$ & $66(57.9)$ & $5(26.3)$ & \\
\hline III & $38(5.9)$ & $14(51.9)$ & $43(37.7)$ & $3(15.8)$ & \\
\hline IV & $7(1.1)$ & $0(0.0)$ & $5(4.4)$ & $0(0.0)$ & \\
\hline MR-proANP, pmol// (median [IQR]) & 65 [42-99] & $46[32-56]$ & 124 [89-202] & 125 [94-209] & $<0.001$ \\
\hline LDL cholesterol, mmol/I (median [IQR]) & $2.0[1.5-2.6]$ & $2.1[1.8-2.6]$ & $1.9[1.6-2.3]$ & $2.1[1.8-2.9]$ & 0.468 \\
\hline Creatinine, $\mu \mathrm{mol} / \mathrm{I}$ (median [IQR]) & $77.0[65.0-93.0]$ & $69.5[60.3-76.8]$ & $97.0[77.0-123.0]$ & $78.0[66.5-96.0]$ & $<0.001$ \\
\hline Albuminuria, n (\%) & $153(23.7)$ & $5(18.5)$ & $37(32.5)$ & $6(31.6)$ & 0.077 \\
\hline $\mathrm{HbA} 1 \mathrm{c}, \mathrm{mmol} / \mathrm{mol}$ (median [IQR]) & $55.0[48.0-65.0]$ & $55.0[48.5-71.0]$ & $54.0[48.0-67.0]$ & $53.0[44.5-69.0]$ & 0.777 \\
\hline Hemoglobin, mmol/L (mean (SD)) & $8.7(0.9)$ & $8.4(0.8)$ & $8.1(1.0)$ & $8.8(0.9)$ & $<0.001$ \\
\hline \multicolumn{6}{|l|}{ Treatment with } \\
\hline Metformin, n (\%) & $488(75.5)$ & $17(63.0)$ & $64(56.1)$ & $11(57.9)$ & $<0.001$ \\
\hline Dipeptidyl peptidase-4 inhibitor, n (\%) & $69(10.7)$ & $4(14.8)$ & $9(7.9)$ & $1(5.3)$ & 0.586 \\
\hline Sulfonylurea, n (\%) & $106(16.4)$ & $2(7.4)$ & $16(14.0)$ & $5(26.3)$ & 0.335 \\
\hline Glucagon-like peptide-1 receptor agonist, n (\%) & $155(24.0)$ & $7(25.9)$ & $18(15.8)$ & $3(15.8)$ & 0.222 \\
\hline Insulin, n (\%) & $275(42.6)$ & $17(63.0)$ & $68(59.6)$ & $13(68.4)$ & $<0.001$ \\
\hline Beta-blocker, n (\%) & $129(20.0)$ & $5(18.5)$ & $54(47.4)$ & $10(52.6)$ & $<0.001$ \\
\hline ACE inhibitor, n (\%) & $256(39.6)$ & $6(22.2)$ & $37(32.5)$ & $10(52.6)$ & 0.081 \\
\hline Angiotensin-II receptor blocker, n (\%) & $241(37.3)$ & $10(37.0)$ & $56(49.1)$ & $7(36.8)$ & 0.123 \\
\hline Calcium channel blocker, n (\%) & $200(31.0)$ & $10(37.0)$ & $44(38.6)$ & $5(26.3)$ & 0.364 \\
\hline Diuretics ${ }^{\mathrm{a}}, \mathrm{n}(\%)$ & $297(46.0)$ & $7(25.9)$ & $77(67.5)$ & $11(57.9)$ & $<0.001$ \\
\hline Statins, n (\%) & $508(78.6)$ & $22(81.5)$ & $88(77.2)$ & $12(63.2)$ & 0.419 \\
\hline Anti-platelet therapy, n (\%) & $423(65.5)$ & $16(59.3)$ & $90(78.9)$ & $15(78.9)$ & 0.019 \\
\hline
\end{tabular}

For patients with $\mathrm{HFpEF}$, stratification according to low $(<60 \mathrm{pmol} / \mathrm{l})$ or high MR-proANP level is presented. $\mathrm{p}$-value for comparison of all four groups

$A C E$, angiotensin converting enzyme; bpm, beats per minute; $\mathrm{HbA1c}$, glycated hemoglobin; $\mathrm{HFpEF}$, heart failure with preserved ejection fraction; $\mathrm{HFrEF}$, heart failure with reduced ejection fraction; IQR, interquartile range; LDL, low density lipoprotein; $\mathrm{mm} \mathrm{Hg}$, millimeters of mercury; MR-proANP, mid-regional pro-atrial natriuretic peptide; sd, standard deviation; T2D, type 2 diabetes mellitus

a Include loop diuretics, thiazides, or mineralocorticoid receptor antagonists

increase in NRI of $27.1 \%$ [7.6-46.6], $\mathrm{p}=0.007$, and adding HF status showed an NRI of $32.8 \%$ [13.5-52.0], $\mathrm{p}<0.001$.
Associations between MR-proANP levels and echocardiographic measures

As supplemental analyses, the associations between MR-proANP levels and the echocardiographic measures were analyzed in univariable and multivariable 


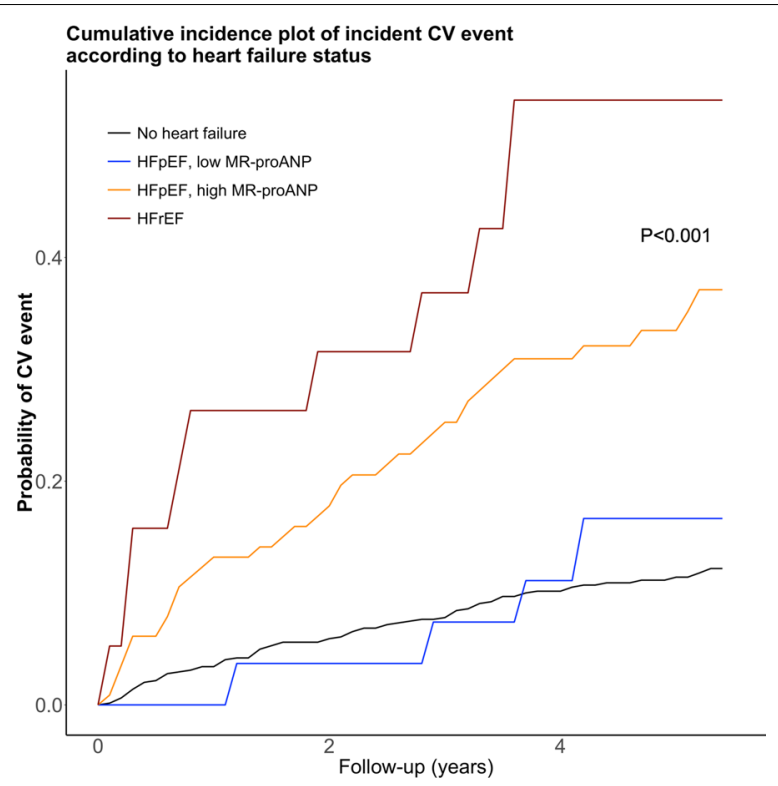

Fig. 1 Cumulative incidence plot of incident cardiovascular events according to HF status. For patients with HFpEF, stratification according to low ( $<60 \mathrm{pmol} / \mathrm{l})$ or high MR-proANP level is presented. CV, cardiovascular; HFpEF, heart failure with preserved ejection fraction; HFrEF, heart failure with reduced ejection fraction; MR-proANP, mid-regional pro-atrial natriuretic peptide

analyses (Additional file 1: Table S2). In the multivariable analyses, MR-proANP was significantly associated with left ventricular mass index, left atrial volume index, the $\mathrm{E} / \mathrm{A}$ ratio, the $\mathrm{E} / \mathrm{e}^{\prime}$ mean ratio, and with measures of left ventricular systolic function.

\section{Discussion}

In this large cohort of patients with T2D in an outpatient, non-acute setting, we found that; (1) higher MR-proANP levels were highly associated with incident CV events; (2) MR-proANP levels effectively stratified patients with symptomatic HFpEF and predicted the outcome of incident $\mathrm{CV}$ events during almost five years of follow-up; (3) patients with symptomatic HFpEF and high MRproANP had an increased risk of $\mathrm{CV}$ events compared to patients with HFpEF without elevated MR-proANP and compared to patients without HF; (4) adding MRproANP as a continuous variable or adding HF status to a model with clinical risk markers significantly reclassified $27.1 \%$ and $32.8 \%$ respectively, but only resulted in limited improvements in C-statistics; (5) finally, MR-proANP was associated with key echocardiographic measures of both diastolic-including left atrial volume index-and systolic function.

In patients with HF, natriuretic peptides are established diagnostic and prognostic biomarkers, and also carry relevant prognostic information in other cardiovascular diseases [7, 16]. Natriuretic peptides act as a surrogate measure of the underlying left ventricular dysfunction in HF [17]. The ventricular dysfunction causes volume expansion and/or pressure overload, leading to increased stress in the atrial and ventricular walls and thereby an increased release of natriuretic peptides. Moreover, natriuretic peptides have also been associated with metabolic changes potentially modulating their property as prognostic markers beyond the indication of ventricular dysfunction [18]. In some series of patients with HF ( $22 \%$ with concomitant T2D), the more
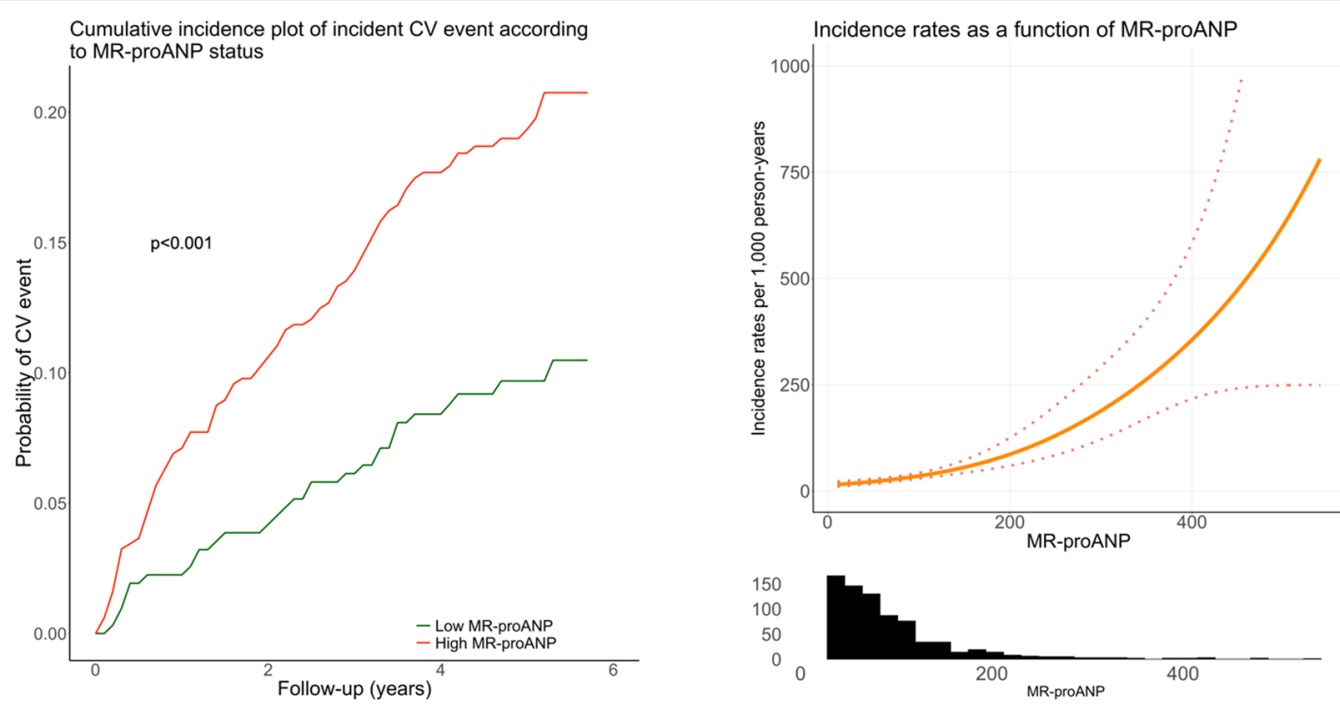

Fig. 2 Cumulative incidence plot of incident CV event and incidence rates according to MR-proANP level. CV, cardiovascular; MR-proANP, mid-regional pro-atrial natriuretic peptide 


$\begin{array}{lll} & \text { n/events } & \text { HR }(\mathbf{9 5} \% \mathbf{C I}) \\ \begin{array}{l}\text { Univariable } \\ \text { log2(MR-proANP) }\end{array} & \\ \text { No heart failure } & & 2.09(1.73-2.52)^{* * *} \\ \text { HFpEF, low MR-proANP } & 646 / 74 & 1.00 \\ \text { HFpEF, high MR-proANP } & 27 / 4 & 1.30(0.48-3.56) \\ \text { HFrEF } & 114 / 38 & 3.47(2.34-5.13)^{* * *} \\ & 19 / 10 & 6.25(3.23-12.11)^{* * *} \\ \text { Multivariable } & & \\ \text { log2(MR-proANP) } & & 1.71(1.34-2.18)^{\star \star *} \\ \text { No heart failure } & & 1.00 \\ \text { HFpEF, low MR-proANP } & 611 / 68 & 2.18(0.78-6.14) \\ \text { HFpEF, high MR-proANP } & 24 / 4 & 2.56(1.64-4.00)^{* * *} \\ \text { HFrEF } & 109 / 36 & 3.32(1.64-6.74)^{* * *} \\ & 19 / 10 & \end{array}$

\section{CV event}

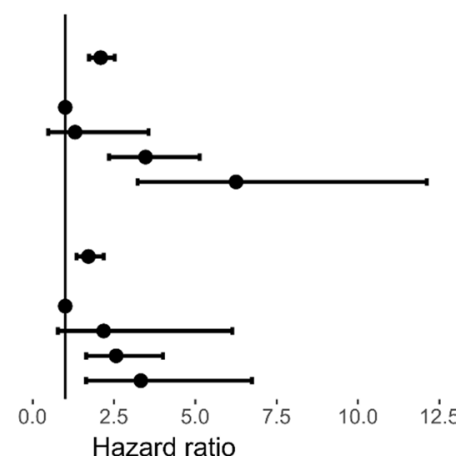

Fig. 3 Forest plot according to HF status, in the univariable analysis and in the multivariable model. ${ }^{* * *} p<0.001 . C V$, cardiovascular; HFpEF, heart failure with preserved ejection fraction; HFrEF, heart failure with reduced ejection fraction; MR-proANP, mid-regional pro-atrial natriuretic peptide

Table 2 Model performance

\begin{tabular}{|c|c|c|c|}
\hline & C-statistics & Net reclassification index & $\mathrm{p}$-value \\
\hline \multicolumn{4}{|l|}{ MR-proANP } \\
\hline Continuous variable (univariable) & $0.66(0.61-0.71)$ & & \\
\hline Cut-off 60 pmol// (univariable) & $0.57(0.54-0.62)$ & & \\
\hline \multicolumn{4}{|l|}{ Multivariable analyses } \\
\hline Model 1 & $0.72(0.68-0.78)$ & & \\
\hline Model 1 + MR-proANP (continuous) & $0.74(0.69-0.78)$ & $27.1 \%(7.6-46.6)$ & 0.007 \\
\hline Model 1 + MR-proANP (Cut-off 60 pmol/l) & $0.73(0.68-0.77)$ & $12.1 \%(-7.0-0.31)$ & 0.22 \\
\hline \multicolumn{4}{|l|}{ Heart failure status } \\
\hline Heart failure status (univariable) & $0.63(0.58-0.67)$ & & \\
\hline Model $1+$ heart failure status & $0.75(0.71-0.80)$ & $32.8 \%(13.5-52.0)$ & $<0.001$ \\
\hline
\end{tabular}

Model 1 includes age, sex, duration of diabetes, known CV disease, SBP $>170 \mathrm{mmHg}$, BMI and albuminuria

The multivariable Cox proportional hazards model (Model 1) with NRI when adding MR-proANP as a continuous or dichomized variable or when adding heart failure status. For reference, the univariable Cox proportional hazards models with MR-proANP as a continuous or dichotomized variable, and with heart failure status are shown

NRI, net reclassification index; MR-proANP, Mid-regional pro-atrial natriuretic peptide; CV, cardiovascular; SBP, systolic blood pressure; BMI, body mass index

recently developed biomarker MR-proANP has outperformed the extensively studied biomarker NT-proBNP in the prediction of mortality, [19] and some data indicate that MR-proANP may be more sensitive and specific than NT-proBNP in diagnosing HFpEF [20]. In patients with T2D, the most common co-existing conditions that cause HF is coronary artery disease and hypertension, [21] and MR-proANP has previously been shown to be independently associated with CV mortality in both primary and secondary care patients with T2D [22, 23]. In 1100 outpatients with T2D prospectively followed in primary care in the Netherlands, MR-proANP as a continuous variable was independently associated with both $\mathrm{CV}$ and all-cause mortality during a follow-up of 10 years with adjusted HRs of 2.42 [1.74-3.38] and 2.23 [1.782.79], respectively, in a multivariable model adjusted for age, sex, smoking, BMI, SBP, duration of diabetes, serum creatinine level, cholesterol-to-high-density lipoprotein ratio, macrovascular complications, albuminuria, and the use of lipid-lowering and anti-hypertensive medications [23]. Similarly, in a prospective cohort of more than 700 outpatients with T2D in secondary care in Austria, a one standard deviation increase in MR-proANP was associated with a 1.85 -fold [1.49-2.30] increase in the risk of the composite outcome of unplanned hospitalization for $\mathrm{CV}$ disease or death during a median follow-up of 15 months, in a multivariable model adjusted for NYHA functional class, age, serum creatinine level, low-density lipoprotein level, level of serum triglycerides, hemoglobin A1c, SBP and BMI [22]. In addition to key differences compared with the present study in characteristics of the cohorts, follow-up times and definitions of outcome, no stratification according to HF status based on echocardiography was performed in these previous studies. 
Stratification according to HF status enables the opportunity to link the MR-proANP levels to the underlying changes in cardiac structure seen in HF patients. In outpatients with T2D, HFrEF is infrequent and can be ruled out with a low MR-proANP level, [2] which is important as therapies have been shown to reduce both morbidity and mortality in patients with HFrEF irrespective of concomitant T2D [4]. Conversely, HFpEF is frequent in outpatients with T2D, [2] and it has been hypothesized that T2D-related processes can cause HFpEF by direct effects on cardiac structure, $[24,25]$ and these changes in cardiac structure have been shown to be associated with $\mathrm{CV}$ events and all-cause mortality [5]. In a recent study, in which $37 \%$ had concomitant T2D, MR-proANP was associated with clinical outcomes in HFpEF, not in HFrEF, supporting the use of MR-proANP as a prognostic marker in patients with HFpEF [9]. Also, the use of MR-proANP in patients with HFpEF is supported by the current data, with a strong association between MRproANP levels and key echocardiographic measures of diastolic-including left atrial volume index-and systolic function which are impaired in these patients. Based on the prognostic data in this large cohort of outpatients with T2D, an MR-proANP level of $60 \mathrm{pmol} / \mathrm{l}$ can support the diabetologist in identifying patients with HFpEF and increased risk of $\mathrm{CV}$ events, thus acting as a guide to identify patients for further cardiac diagnostic work up; patients with dyspnea and an MR-proANP of $60 \mathrm{pmol} / \mathrm{l}$ or greater should be referred for an echocardiogram. At present, the identification of patients with HFpEF has no therapeutic consequence as no effective therapies exist, [26] but patients with HFpEF and increased risk of CV events are candidates for ongoing clinical trials.

The used definition of HFpEF in the present study was prespecified based on the current knowledge in the field. However, as no definitive consensus on the definition of HFpEF exists across guidelines and for inclusion in clinical trials, other definitions could have been valid, and this should be kept in mind when comparing the present results with other studies on HFpEF. Strengths of the present study include the comprehensive characterization of the patients at baseline including echocardiographic assessment. The MR-proANP levels were analyzed in a single batch, consequently reducing potential analytical variability, using a contemporary assay. As the aim was to specifically generate data on MR-proANP, no conclusions regarding potential differences to B-type natriuretic peptides can be made from the present data. The completeness of the registered CV events in the Danish national registries diminishes the possible misclassification of events. Conclusions regarding differences in outcomes between the groups are limited due to the low number of patients in the group with HFpEF and low
MR-proANP and in the group with HFrEF, and larger studies are needed to investigate these possible differences. Additionally, the findings of this study should be tested in other cohorts to ascertain their robustness. The results of the present study cannot be extrapolated to non-diabetic patients or to patients with T2D and atrial fibrillation. Extrapolation to other races than Caucasians should be done with caution. Moreover, despite adjustments for clinically important confounders in the multivariable analyses, the risk of other factors confounding the results is still possible and should be kept in mind when interpreting the results of the present study. A strict cost-benefit analysis should be performed before implementation of routine MR-proANP measurements in the clinical setting.

\section{Conclusions}

In conclusion, our study demonstrates that MR-proANP levels were highly associated with incident cardiovascular events in outpatients with type 2 diabetes and that patients with HFpEF and high MR-proANP levels had a higher risk for cardiovascular events compared to patients with HFpEF without elevated MR-proANP and compared to patients without heart failure. The present findings support the use of MR-proANP as a test in outpatients with type 2 diabetes and support the inclusion of MR-proANP in the definition of HFpEF from a prognostic point-of-view.

\section{Supplementary information}

Supplementary information accompanies this paper at https://doi. org/10.1186/s12933-020-01155-9.

Additional file 1: Table S1. Competing risk analyses with Fine-Gray method with all-cause mortality as competing risk to CV event. Table S2: Associations between MR-proANP and echocardiographic measures.

\section{Abbreviations}

ANP: A-type natriuretic peptide; BMI: Body mass index; BNP: B-type natriuretic peptide; CfD: Center for Diabetes research, Herlev-Gentofte University Hospital; Cl: Confidence interval; CV: Cardiovascular; E: Early diastolic mitral inflow velocity; $\mathrm{e}_{\text {septal: }}$ Early diastolic septal annular velocity; eGFR: Estimated glomerular filtration rate; ESC: European society of cardiology; HF: Heart failure; HFpEF: Heart failure with preserved ejection fraction; HFrEF: Heart failure with reduced ejection fraction; HR: Hazard ratio; IQR: Interquartile range; LV: Left ventricular; LVEF: Left ventricular ejection fraction; MR-proANP: Midregional pro-atrial natriuretic peptide; NRI: Net reclassification index; NT-ANP: N-terminal ANP; NT-proBNP: N-terminal pro-B type natriuretic peptide; NYHA: New York Heart Association; SBP: Systolic blood pressure; sd: Standard deviation; SDCC: Steno Diabetes Center Copenhagen; T2D: Type 2 diabetes.

\section{Acknowledgements}

Not applicable.

Authors' contributions

JJ designed and performed data analysis and wrote the first draft of the manuscript. PGJ designed and performed data collection, designed and performed 
data analysis. MS designed data analysis. MTJ performed data collection. All authors revised the manuscript for important intellectual content. All authors read and approved the final manuscript.

\section{Funding}

Thermo Fisher Scientific (Germany) funded the MR-proANP kits. The sponsor had no influence on design of the study, collection, analysis or interpretation of the data, manuscript preparation or on the decision to submit the manuscript for publication.

\section{Availability of data and materials}

The datasets used and/or analyzed during the current study are available from the corresponding author on reasonable request.

\section{Ethics approval and consent to participate}

All participants provided written informed consent and the study was approved by the Danish National Committee on Biomedical Research Ethics (amendment to protocol no. H-3-2009-139).

\section{Consent for publication}

Not applicable.

\section{Competing interests}

M.S. reports lecture fees received from Novo Nordisk and Boehringer Ingelheim. M.T.J. has served as consultant, on advisory boards, or invited speaker for Astra Zeneca, Novo Nordisk, Novartis, and General Electric. H.U.A. owns stocks in Novo Nordisk. HUA is on advisory boards for Novo Nordisk, Abbott and Astra Zeneca and has received a lecture fee from Nordic Infucare. P.R. reports grants from and shares in Novo Nordisk, service on advisory boards for Astra Zeneca, Bayer, Boehringer Ingelheim, Gilead, Eli Lilly, Novo Nordisk, Merck, Mundipharma, Astellas, Sanofi Aventis (all fees to his institution) and receipt of lecture fees from Bayer, Astra Zeneca and Eli Lilly (to his institution). T.V. reports grants/personal fees/unrestricted research grants from Novo Nordisk, Boehringer, Sanofi, Eli Lilly, MSD/Merck, BMS, Sun Pharmaceuticals, Mundipharma and Amgen. P.G.J. reports lecture fees from Novo Nordisk and Astra Zeneca. The authors have no other relevant conflicts of interest.

\section{Author details}

1 Department of Cardiology, Herlev and Gentofte Hospital, Borgmester Ib Juuls Vej 1, 2730 Herlev, Denmark. ${ }^{2}$ Department of Endocrinology, Rigshospitalet, Blegdamsvej 9, 2100 Copenhagen, Denmark. ${ }^{3}$ Department of Endocrinology, Herlev and Gentofte Hospital, Borgmester Ib Juuls Vej 1, 2730 Herlev, Denmark. ${ }^{4}$ Steno Diabetes Center Copenhagen, Niels Steensens Vej 2, 2820 Gentofte, Denmark. ${ }^{5}$ Department of Cardiology, Copenhagen University Hospital Amager-Hvidovre Hospital, Kettegård Alle 30, 2650 Hvidovre, Denmark. ${ }^{6}$ Faculty of Health and Medical Sciences, Copenhagen University, Blegdamsvej 3B, 2200 Copenhagen, Denmark.

Received: 9 July 2020 Accepted: 10 October 2020 Published online: 16 October 2020

\section{References}

1. Bell DSH, Goncalves E. Heart failure in the patient with diabetes: Epidemiology, aetiology, prognosis, therapy and the effect of glucose-lowering medications. Diabetes Obes Metab. 2019:21:1277-90.

2. Jensen J, Schou M, Kistorp C, Faber J, Hansen TW, Jensen MT, et al. Prevalence of heart failure and the diagnostic value of MR-proANP in outpatients with type 2 diabetes. Diabetes Obes Metab. 2019;21:736-40.

3. Zakeri R, Cowie MR. Heart failure with preserved ejection fraction: controversies, challenges and future directions. Heart. 2018;104:377-84.

4. Ponikowski P, Voors AA, Anker SD, Bueno H, Cleland JGF, Coats AJS, et al. 2016 ESC Guidelines for the diagnosis and treatment of acute and chronic heart failure: the task force for the diagnosis and treatment of acute and chronic heart failure of the European Society of Cardiology (ESC) Developed with the special contribution of the Heart Failure Association (HFA) of the ESC. Eur Heart J. 2016:37:2129-200.

5. Jorgensen PG, Schou M, Biering-Sorensen T, Mogelvang R, Fritz-Hansen T, Vilsboll T, et al. An echocardiographic substrate for dyspnea identifies high risk patients with type 2 diabetes. Int J Cardiol. 2019;289:119-24.
6. Tanase DM, Radu S, Al Shurbaji S, Baroi GL, Florida Costea C, Turliuc MD, et al. Natriuretic peptides in heart failure with preserved left ventricular ejection fraction: from molecular evidences to clinical implications. Int J Mol Sci. 2019;20:2629

7. Mueller C, McDonald K, de Boer RA, Maisel A, Cleland JGF, Kozhuharov $\mathrm{N}$, et al. Heart failure association of the European Society of Cardiology practical guidance on the use of natriuretic peptide concentrations. Eur J Heart Fail. 2019;21:715-31.

8. Maisel A, Mueller C, Nowak R, Peacock WF, Landsberg JW, Ponikowski P, et al. Mid-region pro-hormone markers for diagnosis and prognosis in acute dyspnea: results from the BACH (Biomarkers in Acute Heart Failure) trial. J Am Coll Cardiol. 2010;55:2062-76.

9. Putko BN, Savu A, Kaul P, Ezekowitz J, Dyck JR, Anderson TJ, et al. Left atrial remodelling, mid-regional pro-atrial natriuretic peptide, and prognosis across a range of ejection fractions in heart failure. Eur Heart J Cardiovasc Imag 2020.

10. Smith JG, Newton-Cheh C, Almgren P, Struck J, Morgenthaler NG, Bergmann A, et al. Assessment of conventional cardiovascular risk factors and multiple biomarkers for the prediction of incident heart failure and atrial fibrillation. J Am Coll Cardiol. 2010;56:1712-9.

11. Gohar A, Rutten FH, den Ruijter H, Kelder JC, von Haehling S, Anker SD, et al. Mid-regional pro-atrial natriuretic peptide for the early detection of non-acute heart failure. Eur J Heart Fail. 2019;21:1219-27.

12. Jensen MT, Sogaard P, Andersen HU, Bech J, Hansen TF, Galatius S, et al. Prevalence of systolic and diastolic dysfunction in patients with type 1 diabetes without known heart disease: the Thousand \& 1 Study. Diabetologia. 2014;57:672-80.

13. Jorgensen PG, Jensen MT, Mogelvang R, von Scholten BJ, Bech J, FritzHansen $\mathrm{T}$, et al. Abnormal echocardiography in patients with type 2 diabetes and relation to symptoms and clinical characteristics. Diab Vasc Dis Res. 2016;13:321-30.

14. Jorgensen PG, Jensen MT, Mogelvang R, Fritz-Hansen T, Galatius S, Biering-Sorensen T, et al. Impact of type 2 diabetes and duration of type 2 diabetes on cardiac structure and function. Int J Cardiol. 2016;221:114-21.

15. Jorgensen PG, Biering-Sorensen T, Mogelvang R, Fritz-Hansen T, Vilsboll T, Rossing P, et al. Predictive value of echocardiography in Type 2 diabetes. Eur Heart J Cardiovasc Imaging. 2019;20:687-93.

16. Wong YK, Cheung CYY, Tang CS, Hai JSH, Lee CH, Lau KK, et al. High-sensitivity troponin I and B-type natriuretic peptide biomarkers for prediction of cardiovascular events in patients with coronary artery disease with and without diabetes mellitus. Cardiovasc Diabetol. 2019;18:171.

17. Luchner A, Burnett JC Jr, Jougasaki M, Hense HW, Heid IM, Muders F, et al. Evaluation of brain natriuretic peptide as marker of left ventricular dysfunction and hypertrophy in the population. J Hypertens. 2000;18:1121-8.

18. Masuch A, Pietzner M, Bahls M, Budde K, Kastenmuller G, Zylla S, et al. Metabolomic profiling implicates adiponectin as mediator of a favorable lipoprotein profile associated with NT-proBNP. Cardiovasc Diabetol. 2018;17:120.

19. Moertl D, Berger R, Struck J, Gleiss A, Hammer A, Morgenthaler NG, et al. Comparison of midregional pro-atrial and B-type natriuretic peptides in chronic heart failure: influencing factors, detection of left ventricular systolic dysfunction, and prediction of death. J Am Coll Cardiol. 2009;53:1783-90.

20. Cui K, Huang W, Fan J, Lei H. Midregional pro-atrial natriuretic peptide is a superior biomarker to $\mathrm{N}$-terminal pro-B-type natriuretic peptide in the diagnosis of heart failure patients with preserved ejection fraction. Medicine. 2018;97:e12277.

21. Seferovic PM, Petrie MC, Filippatos GS, Anker SD, Rosano G, Bauersachs J, et al. Type 2 diabetes mellitus and heart failure: a position statement from the heart failure association of the European Society of Cardiology. Eur J Heart Fail. 2018;20:853-72.

22. Maier C, Clodi M, Neuhold S, Resl M, Elhenicky M, Prager R, et al. Endothelial markers may link kidney function to cardiovascular events in type 2 diabetes. Diabetes Care. 2009;32:1890-5.

23. van Hateren KJ, Landman GW, Kleefstra N, Groenier KH, Struck J, Navis GJ, et al. The midregional fragment of pro-A-type natriuretic peptide, blood pressure, and mortality in a prospective cohort study of patients with type 2 diabetes (ZODIAC-25). Diabetes Care. 2013;36:1347-52. 
24. Seferovic PM, Paulus WJ. Clinical diabetic cardiomyopathy: a twofaced disease with restrictive and dilated phenotypes. Eur Heart J. 2015;36(1718-27):27a-27c.

25. Jensen MT, Fung K, Aung N, Sanghvi MM, Chadalavada S, Paiva JM, et al. Changes in cardiac morphology and function in individuals with diabetes mellitus: The UK biobank cardiovascular magnetic resonance substudy. Circ Cardiovasc Imaging. 2019;12:e009476.

26. Lam CSP, Voors AA, de Boer RA, Solomon SD, van Veldhuisen DJ. Heart failure with preserved ejection fraction: from mechanisms to therapies. Eur Heart J. 2018;39:2780-92.

\section{Publisher's Note}

Springer Nature remains neutral with regard to jurisdictional claims in published maps and institutional affiliations.
Ready to submit your research? Choose BMC and benefit from:

- fast, convenient online submission

- thorough peer review by experienced researchers in your field

- rapid publication on acceptance

- support for research data, including large and complex data types

- gold Open Access which fosters wider collaboration and increased citations

- maximum visibility for your research: over $100 \mathrm{M}$ website views per year

At BMC, research is always in progress.

Learn more biomedcentral.com/submissions 